\title{
Introduction to the special issue on information technologies in logistics and service science
}

\author{
Honglu Liu • Guowei Hua
}

Published online: 22 June 2013

(c) Springer Science+Business Media New York 2013

Information technology has been playing an increasingly important role in every walk of life since the third industrial revolution. In order to realize more efficient and smarter manufacturing, working and living spaces, enabling information technologies such as cloud computing and big data are emerging themes among global research scholars.

Logistics is a typical sector that is regularly enhanced by every revolution of information technology. On the one hand, information technology makes more kinds of information available in supply chains, many of which used not to be available. Also, technology accelerates the information sharing across supply chains, and among supply chain partners. For instance, radio frequency identification devices (RFID) and wireless sensor networks (WSN) as well as other enabling technologies bring the supply chain visibility, realizing "real-time logistics" by sharing more immediate information about products and goods.

To achieve satisfied service users in cyber space or daily digital life, proper use of information technology is of tantamount importance as the availability of data grows exponentially. Many research projects on data mining, big data analysis and cloud computing, or related technologies,

\section{H. Liu $(\square)$}

School of Traffic and Transportation, Beijing Jiaotong

University, Beijing 100044, China

e-mail: hlliu@bjtu.edu.cn

G. Hua

School of Economics and Management, Beijing Jiaotong University, Beijing 100044, China

e-mail: huaguowei@gmail.com

G. Hua

Department of Industrial Engineering and Operations Research, University of California, Berkeley, CA 94720, USA could effectively facilitate qualified service by catching and providing valuable information to individuals from an ocean of data.

This special issue includes 4 articles on different aspects of information technologies adaptable in logistics or service science. The first paper, "A resource integration approach for HTML5 mobile applications" by Zhu et al., focuses on an Internet information integration method. The authors propose an integration approach for mobile web applications, which can assist application service providers to provide HTML5-based mobile hybrid applications for various types of mobile ends. And correspondent prototype platform that constructs more flexible mechanism of integration is developed in the article.

The paper entitled "An Inter-PAN Mobility Support Scheme for IP-based Wireless Sensor Networks and its Applications" by Shang et al. presents a novel mobile management scheme used in internet protocol (IP) based WSN. This scheme is able to support many exciting applications such as healthcare system, logistics and storage, as demonstrated by the authors.

In order to deal with the problem of unbalanced data classification and asymmetry misclassification cost in loan default discrimination problem, Cao et al. in their paper "A loan default discrimination model using cost-sensitive support vector machine improved by particle swarm optimization (PSO)" propose a novel PSO-CS-SVM model that hybridizes the PSO and cost sensitive support vector machine (CSSVM). This is a research on data classification technology, available to underpin high quality finical service.

In the last paper "A new evolution model for B2C e-commerce market", Tian et al. study B2C e-commerce market from the view of network. A new proposed model reveals a "bubble-stable" evolutionary process, which is correspondent with real e-commerce market from an initial 
network to a scale-free one, and demonstrates that unfair competition among websites may be the reason for the formation of structure.

We hope that this special issue will serve our Information Technology and Management readers as an avenue to gain a new perspective on information technology in logistics and service science. The efforts that the authors made and the contributions of the reviewers are especially notable. We would especially like to thank the Co-Editor in Chief, Varghese Jacob, Erik Rolland and Ray Patterson, and the Journal Manager, Deepika Jothinathan for their strong support, which made the special issue possible. 